\title{
Observation of enhanced fluctuation diamagnetism in lanthanum superconductors with dilute magnetic impurities
}

\author{
Félix Soto, Lucía Cabo, Jesús Mosqueira, \\ Manuel V. Ramallo, José A. Veira and Félix Vidal \\ Laboratorio de Baixas Temperaturas e Superconductividade, ${ }^{1}$ \\ Departamento de Física da Materia Condensada, \\ Universidade de Santiago de Compostela, E15782 Spain.
}

\begin{abstract}
The fluctuation-induced diamagnetism $\Delta M$, associated with the presence of precursor Cooper pairs in the normal state, has been measured in lanthanum with dilute magnetic $(\mathrm{Pr})$ and nonmagnetic $(\mathrm{Lu})$ impurities. It is found that while for pure La and La-Lu alloys $\Delta M$ agrees, as expected, with the theoretical predictions, it is much larger for La-Pr alloys (around a factor 5 for La- 2 at.\%Pr). These results suggest the existence of an indirect contribution to $\Delta M$ arising from the interaction between fluctuating Cooper pairs and magnetic impurities.
\end{abstract}

\footnotetext{
${ }^{1}$ Unidad Asociada al Instituto de Ciencia de Materiales de Madrid, CSIC, Spain
} 
The magnetic susceptibility measurements of Matthias and coworkers[1] showing the decrease of the superconducting transition temperature of lanthanum with dilute magnetic rare earth impurities, and the explanation of these effects by Abrikosov and Gor'kov[2] in terms of pair-breaking, opened about 45 years ago one of the still at present most interesting and studied issues of correlated electron systems: the interplay between magnetism and superconductivity. 3, 4. A natural question directly related to these pioneering results but to our knowledge not yet addressed until now is how the magnetic impurities will affect the precursor diamagnetism associated with the presence of fluctuating Cooper pairs created above any superconducting transition by the unavoidable thermal agitation. In fact, this fluctuation-induced diamagnetism above the superconducting transition may be used as an unique probe to study the competition between pairing correlations and magnetic order without entering into the fully-superconducting state, which would hide the response of the magnetic ions to external fields.

We present in this Letter measurements of the fluctuation-induced diamagnetism, $\Delta M \equiv M(T)-M_{B}(T)$, in La with dilute (up to 2 at.\%) magnetic (Pr) and nonmagnetic (Lu) impurities. Here $M(T)$ and $M_{B}(T)$ are the measured and, respectively, the background or bare magnetizations. It is found that while for pure La and La-Lu alloys $\Delta M$ agrees with the calculations on the grounds of the Gaussian-Ginzburg-Landau (GGL) approach for isotropic 3D superconductors [5, 6, 7, 8, the $\Delta M$ amplitude is much larger in the La-Pr alloys (about 500\% for impurity concentrations of 2 at.\%). The results for La-Lu alloys just confirm previous experiments in other dirty low- $T_{C}$ superconductors [6, 7, 8, and they may be easily understood by just assuming that the fluctuating Cooper pairs in the normal state are also protected, as the Cooper pairs below the superconducting critical temperature $T_{C}$, by the Anderson theorem for symmetric perturbations. [10] These results in La-Lu superconductors provide then a crucial check of the reliability of our measurements of $\Delta M$ in the lanthanum-based alloys. In contrast with these conventional results in La-Lu alloys, the $\Delta M$-enhancement in presence of magnetic ions suggests not only that the fluctuating Cooper pairs in conventional (singlet $s$-wave pairing) BCS superconductors are very robust to antisymmetric perturbations but also the existence of an indirect contribution to $\Delta M$ arising from the interaction between Cooper pairs and magnetic impurities. These results may have implications in other scenarios where superconducting fluctuations and magnetic order 
coexist [3, 4], in particular when the superconductivity is magnetically mediated [1], perhaps including the high- $T_{C}$ cuprates 4 .

In addition to its interest as a natural extension of the seminal experiments of Matthias and coworkers, [1] our choice of the magnetization to study the interplay between fluctuating Cooper pairs in the normal state and magnetic impurities was motivated by the fact that, as known since the early experiments of Tinkham and coworkers in low- $T_{C}$ superconductors without magnetic impurities, [6, 7] the fluctuation-induced magnetization probably is the best observable to probe the superconducting fluctuations in any isotropic bulk (3D) superconductor. In contrast with most of the effects of the superconducting fluctuations on other observables, $|\Delta M|$ increases not only with the density of fluctuating Cooper pairs but also with their size, i.e., with the superconducting coherence length amplitude (extrapolated to $T=0 \mathrm{~K}$ ), $\xi(0)$. As a consequence, $\left|\Delta M / M_{B}\right|$ may take relatively high values at easily accessible temperature-distances from the transition in isotropic low- $T_{C}$ superconductors, even bigger than in high$T_{C}$ superconductors, due to their much larger $\xi(0)$. [8] In fact, for the superconductors studied in the present Letter we will obtain values of $\left|\Delta M / M_{B}\right|$ well comparable (about one order of magnitude smaller in the worst case) with those of optimally-doped $\mathrm{YBa}_{2} \mathrm{Cu}_{3} \mathrm{O}_{7-\delta} \cdot 9$. These experimental advantages appear to be crucial when compared with the difficulties that may arise if one uses other observables, very in particular the electrical conductivity, to study the interplay between superconducting fluctuations and magnetic impurities in low- $T_{C}$ superconductors: mainly in bulk materials, the ratio between the corresponding paraconductivity, $\Delta \sigma$, and the nonfluctuating (background) conductivity, $\sigma_{B}$, may be orders of magnitude smaller than $\left|\Delta M / M_{B}\right|$. In addition, $\sigma_{B}$ may be much more affected than $M_{B}$ by stoichiometric and structural inhomogeneities. These difficulties probably affect deeply the few (to our knowledge) attempts published until now to study through the electrical conductivity the interplay between superconducting fluctuations and magnetic impurities in low- $T_{C}$ superconductors. [12, 13]

The fitness of the La alloys to measure $\Delta M$ in presence of magnetic impurities is mainly due to the fact that these compounds still have a quite large $\xi(0)$, of the order of $200 \AA$. Complementarily, for some magnetic impurities (very in particular Pr), their normal state magnetization is expected to remain relatively moderate, even under impurity concentrations $x$ as important as 2 at. $\%$, the larger impurity concentrations used 
by Matthias and coworkers in their measurements of $T_{C}(x)$ [1]. In addition, at present high-quality La alloys are commercially available. The polycrystalline $\operatorname{La}_{100-x} \operatorname{Pr}_{x}$ and $\mathrm{La}_{100-x} \mathrm{Lu}_{x}$ alloys studied in this Letter, with $0 \leq x \leq 2$, were supplied by Goodfellow and Alfa Aesar, and their impurity concentration was controlled to better than $0.1 \%$. The superconducting transition width, $\Delta T_{C}$, as determined from electrical resistivity and field-cooled magnetic susceptibility measurements remains below $\sim 0.25 \mathrm{~K}$ for all the samples, which confirms their good stoichiometric and structural quality. These measurements, as well as those performed to determine other general characteristics of the samples (very in particular $H_{c 2}(0)$ and then $\xi(0)$ ), are detailed elsewhere 14. Here we present in table 1 some of the main sample parameters and in fig. 1(a) the impurity concentration dependence of $T_{C}$, which for the La-Pr alloys provides a direct measure of the pair-breaking effects induced by the magnetic impurities. These $T_{C}(x)$ data are in agreement with both the results of Matthias and coworkers 1 and the AbrikosovGor'kov approach [2]. The solid line in fig. 1(a) is a fit of this approach, which at low concentrations reduces to $T_{C}(x=0)-T_{C}(x)=\hbar / k_{\mathrm{B}} \tau_{\phi}(x)$, where $\hbar$ is the reduced Planck constant, $k_{\mathrm{B}}$ is the Boltzmann constant and $\tau_{\phi}(x)=\tau_{\phi}(x=1) / x$ is the phase pair-breaking time. This leads to $\tau_{\phi}(x=1) \simeq 3.4 \times 10^{-11} \mathrm{~s}$, which is much larger than the relaxation time of the normal electrons deduced from resistivity measurements [14] $\left(\tau \sim 10^{-14} \mathrm{~s}\right) . T_{C}(x)$ for the alloys with nonmagnetic Lu-impurities is almost constant, the Cooper pairs in these last alloys being protected, as is well known, by the Anderson theorem. 10.

An example of the temperature behaviour above $T_{C}$ of the measured and of the background magnetizations is presented in figs. 1(b) and (c). These measurements were performed under constant magnetic field amplitude (and in the low-field regime, $\left.H \ll H_{c 2}(0)\right)$ with a commercial, SQUID based, magnetometer (Quantum Design's MPMS) and by using cylindrical samples of diameter and height $\sim 5 \mathrm{~mm}$, the maximum volume allowed by our magnetometer. The demagnetizing effects are negligible above $T_{C}$. Other experimental details are similar to those in magnetization measurements in other low- $T_{C}$ superconductors. [8] The solid line in figs. 1(b) and (c) is the background or bare magnetization (over $H$ ), obtained by fitting in the temperature region bounded by $1.5 T_{C} \leq T \leq 3 T_{C}$ the function $M_{B}(T) / H=\chi_{0}+B T+C / T$ (with $\chi_{0}, B$ and $C$ as free parameters). In this region the agreement between $M_{B}(T) / H$ (solid line) and the data is excellent, the maximum deviation being below $0.05 \%$. Let us stress that 
even above this background fitting region, up to $5 T_{C}$, the agreement with the data still remains quite good, the maximum deviation being below 5\%. The above background functionality is expected to be a good approximation for (anti)ferromagnetic materials at temperatures well above the Curie or Néel temperatures $T_{\mathrm{M}},[15$, which in superconductors with diluted magnetic impurities may be estimated through the relationship [16] $T_{\mathrm{M}}(x) \sim T_{C}(x=0)-T_{C}(x)$. In our samples this leads to $T_{\mathrm{M}} \lesssim 0.5 \mathrm{~K} \ll T_{C}$.

From curves similar to those presented in fig. 1(b), we obtain the fluctuation-induced magnetization versus reduced-temperature curves, $\Delta M(\varepsilon)$, where $\varepsilon \equiv \ln \left(T / T_{C}\right)$. In fig. 2(a) we present some examples of these $\Delta M(\varepsilon)$ curves, very in particular those that correspond to the La-Pr alloys. These data are well inside the $\varepsilon$-range where the GGL approach is expected to be applicable. [5, 6, 7, 8, 9] The solid line corresponds to the GGL calculations for isotropic 3D superconductors without magnetic impurities in the zero-field limit, [5, 6, 8]

$$
\frac{\Delta M^{\mathrm{GGL}}(\varepsilon)}{H \xi(0) T}=\frac{-\mu_{0} k_{\mathrm{B}}}{3 \phi_{0}^{2}}\left[\frac{\arctan \sqrt{\frac{\varepsilon^{C}-\varepsilon}{\varepsilon}}}{\sqrt{\varepsilon}}-\frac{\arctan \sqrt{\frac{\varepsilon^{C}-\varepsilon}{\varepsilon^{C}}}}{\sqrt{\varepsilon^{C}}}\right],
$$

where $\varepsilon^{C} \simeq 0.6$ is the BCS value of the cutoff constant [17] and $\phi_{0}$ is the flux quantum. As visible in this figure, the data in pure La and in La-Lu alloys agree with Eq.(1) well within the experimental uncertainties, which are of the order or below $40 \%[18$, an agreement that was already observed in other low- $T_{C}$ superconductors with nonmagnetic impurities. [8] As stressed before, this result may be easily understood by just assuming the applicability also above $T_{C}$ of the immunity predicted by Anderson[10] of the Cooper pairs to symmetric perturbations. So, these last results also show the reliability of our present experiments to probe the superconducting fluctuations above $T_{C}$ in La alloys.

The results of fig. 2(a) show also that in presence of magnetic impurities both the amplitude and the $\varepsilon$-dependence of $\Delta M(\varepsilon) / H \xi(0) T$ differ from the GGL calculations [5], 6. 8], even for the alloy with the lower Pr concentration: The $\Delta M(\varepsilon) / H \xi(0) T$ amplitudes remain much larger, about five times for the La-2 at.\%Pr alloy, and also their reduced-temperature dependence is smoother. As illustrated quantitatively in fig. 2(b), this enhancement is essentially proportional to the magnetic impurity concentration, in striking contrast with the well established and well understood decrease of the su- 
perconducting transition temperature when such a concentration increases [1, 2, 3] (see fig. 1(a)).

In analyzing the experimental findings summarized in figs. 2(a) and (b) we must start by checking if such an strong $\Delta M$-enhancement is just an effect due to $T_{C^{-}}$ inhomogeneities at long-length scales (bigger than $\xi(0)$ ), in turn associated with stoichiometric and structural defects. [19] However, this extrinsic effect is ruled out not only by the high quality of the samples but mainly by the fact that the La-Lu alloys display magnetic and electrical resistivity transition widths, $\Delta T_{C}$, similar to those of their La-Pr counterparts (see table 1). Also, a rounding of the magnetic transition due to local $T_{C}$-inhomogeneities, induced by the magnetic ions (inhomogeneities either isolated [20] or Josephson tunneling-connected 21]), may be discarded because in our La-Pr alloys the mean distances among impurities, $d_{\mathrm{imp}}$, are in the range $12-19 \AA$, which is well below the $\xi(0)$ values $\sim 200 \AA$ (see table 1 ).

The amplification effects associated with a reduction of the superconducting fluctuations' dimensionality (in turn due to a confinement of these fluctuations between the impurity interspaces) may be also discarded. This is illustrated in fig. 2(b), where the dot-dashed line corresponds to the fluctuation magnetization that may be obtained by supposing that the magnetic impurities would allow only 0D superconducting fluctuations occurring in the interspace between magnetic impurities. For such a $\Delta M(\varepsilon)$ in $0 \mathrm{D}$ we used[6] $\Delta M^{0 \mathrm{D}}(\varepsilon)=\left(\xi(0) / d_{\mathrm{imp}} \varepsilon^{1 / 2}\right) \Delta M^{\mathrm{GGL}}(\varepsilon)$.

Another possible amplification effect, opposite in origin to a superconducting fluctuations' confinement, would be that these fluctuations embrace part of the magnetic ions, cancelling their contribution to the magnetization. A crude estimation of these effects may be done by just identifying $\left|\Delta M^{\mathrm{GGL}} / H\right|$ with the effective volume fraction occupied by the evanescent superconducting "droplets". The resulting contribution to the magnetization is then $M_{B} \Delta M^{\mathrm{GGL}} / H$ which, taking into account that in our samples $10^{-4} \lesssim M_{B} / H \lesssim 10^{-3}$ near the transition, leads to a negligible effect. Finally, we also note that a mechanism of internal-field screening different to Meissner-like currents, which involves spin alignment of the quasiparticles composing the Cooper pair, has been recently proposed, for $T<T_{C}$ and noninteracting magnetic impurities. 22. However, when applied to $T>T_{C}$ the resulting change in magnetization is again about three orders of magnitude smaller than our observations. 
In absence of a theoretical approach for the superconducting fluctuations in presence of magnetic impurities, and as a first crude attempt to establish empirically the physical origin of the observed $\Delta M$-enhancement, we have checked if it is possible to separate the measured $\Delta M(\varepsilon)$ in two contributions: the direct GGL Schmidt and Schmid term and an indirect contribution proportional to both the density of fluctuating Cooper pairs and the magnetic impurity concentration. This check has been summarized in figs. 2(a) and 2(b), where the dashed lines were obtained using the empirical expression

$$
\Delta M(\varepsilon)=\Delta M^{\mathrm{GGL}}(\varepsilon)+A H x n_{s}(\varepsilon),
$$

where $A$ is a constant and $n_{s}(\varepsilon)$ is the superfluid density (in dimensionless units) for isotropic 3D superconductors in the zero-field limit, [17.

$$
n_{s}(\varepsilon)=\frac{k_{\mathrm{B}} \mu_{0} \mathrm{e}^{2} T \xi(0)}{\hbar^{2} \sqrt{\varepsilon}}\left[\sqrt{\frac{\varepsilon^{C}-\varepsilon}{\varepsilon}}-\arctan \sqrt{\frac{\varepsilon^{C}-\varepsilon}{\varepsilon}}\right] .
$$

The dashed lines in fig. 2(a) correspond to fits to the La-Pr alloy data of the above $\Delta M(\varepsilon)$ expression, with $A$ as the only free parameter (we constrained the rest of parameters to be compatible with table 1 , and also used $\varepsilon^{C}=0.6$ for all the fits). As it may be seen in this figure, the agreement between the experimental results and Eq.(2) is excellent, for both the amplitude and the $\varepsilon$-behaviour, and this using the same value $A \simeq-0.75$ for all the samples.

The above results lead to two complementary conclusions: First, the presence of the direct GGL Schmidt and Schmid contribution in the La-Pr alloys suggests the robustness of the fluctuating Cooper pairs to dilute magnetic impurities. Although unexpected when compared to the decrease of $T_{C}(x)$ shown in fig. 1(a), this behaviour agrees with recent $\Delta M$-measurements in $\mathrm{Pb}$-In alloys under high reduced-fields, 23 . which also suggests the robustness of the fluctuating Cooper pairs to the antisymmetric nature of an applied magnetic field. Complementarily, the presence of an indirect contribution to $\Delta M(\varepsilon)$ in the La-Pr alloys suggests that the fluctuating Cooper pairs modify the coupling between the magnetic ions. In the case of dilute alloys such a coupling is mediated by the electronic sea (RKKY model). [15, 25] It may be then expected that the changes induced in the electrons' spin susceptibility by the Cooper pair formation will also affect the magnetization due to the magnetic ions. In fact, such an effect was early proposed below $T_{C}$ [24], and the corresponding change in the 
magnetization above $T_{C}$ may be expected to be proportional to both the superfluid density and the concentration of magnetic ions, in agreement with our experimental findings. These results suggest that the energetic balance between the magnetic and the superconducting orders could be shifted by the presence of precursor Cooper pairs. Although these crude ideas need to be confirmed both experimentally and theoretically, the experimental findings presented in this Letter already stress that the superconducting fluctuations may play an unexpected important role in the interplay between magnetism and superconductivity, mainly if the latter is magnetically mediated. 4, 11.

\section{Acknowledgments}

We acknowledge useful conversations and correspondence with J.B. Goodenough and K. Maki on this topic. This work was supported by the CICYT, Spain (grant no. MAT2004-04364), the Xunta de Galicia (PGIDT01PXI20609PR), and Unión Fenosa (220/ 0085-2002). LC acknowledges financial support from Spain's Ministerio de Educación y Ciencia through a FPU grant.

\section{References}

[1] B.T. Matthias, H. Suhl and E. Corenzwit, Phys. Rev. Lett. 1, 92 (1958); R.A. Hein et al., ibid. 2, 500 (1959).

[2] A.A. Abrikosov and L.P. Gor'kov, Sov. Phys. JETP 12, 1243 (1961).

[3] See, e.g., Ø. Fisher, in Ferromagnetic Materials, Vol. 5, edited by K.H.J. Buschow and E.P. Wohlfarth (Elsevier, Amsterdam) 1990, and references therein.

[4] See, e.g., Correlated Electron Systems (Science 288, pp.461-482 (2000)).

[5] H. Schmidt, Z. Phys. 216, 336 (1968); A. Schmid, Phys. Rev. 180, 527 (1969). 
[6] For a review see, e.g., J. Skocpol and M. Tinkham, Repts. Prog. Phys. 38, 1049 (1975); M. Tinkham, Introduction to superconductivity (McGraw-Hill, N.Y.) 1996, chap. 8.

[7] J.P. Gollub, M.R. Beasley, R.S. Newbower and M. Tinkham, Phys. Rev. Lett. 22, 1288 (1969); J.P. Gollub, M.R. Beasley and M. Tinkham, ibid. 25, 1646 (1970); J.P. Gollub et al., Phys. Rev. B 7, 3039 (1973).

[8] J. Mosqueira, C. Carballeira and F. Vidal, Phys. Rev. Lett. 87, 167009 (2001).

[9] See, e.g., F. Vidal and M.V. Ramallo, in The gap symmetry and fluctuations in high-T $T_{C}$ superconductors, edited by J. Bok et al. (Plenum, N.Y.) 1998, p. 443, and references therein.

[10] P.W. Anderson, J. Phys. Chem. Solids 11, 26 (1959).

[11] See, e.g., S.S. Saxena et al., Nature 406, 587 (2000).

[12] E. Spahn and K. Keck, Physica B 165-166, 1357 (1990). The samples measured in this work are $\mathrm{Al}$ films with a magnetic (Ni) covering, and therefore probably are highly inhomogeneous.

[13] P. Lindqvist, A. Nordström and Ö. Rapp, Phys. Rev. Lett. 64, 2941 (1990). As shown in Ref. 19, the anomalies reported here may be easily explained just in terms of extrinsic $T_{C}$-inhomogeneities at long length scales (much larger than $\xi(0))$ and non-uniformly distributed.

[14] F. Soto et al., J. Phys. Chem. Solids (in press). An extended version of this paper may be found in cond-mat/0511033.

[15] See, e.g., J. Crangle, Solid state magnetism (Edward Arnold, London) 1991, chaps. 6 and 7 . The first two $M_{B} / T$ contributions, $\chi_{0}$ and the small $B T$, are usual in materials without magnetic impurities (and were also used in the analyses of $\Delta M$ in low- $T_{C}$ superconductors without magnetic impurities as, e.g., Pb-In alloys [8]). The magnetic impurities, at the low magnetic fields used in this work (well smaller than the saturation fields), will produce additional $M_{B} / T$ contributions $\propto\left(T \pm T_{\mathrm{M}}\right)^{-1}$, which in our $T_{\mathrm{M}} \ll T$ case may be safely approximated as $C / T$. (Note that the correlation of very diluted magnetic impurities will be 
mediated by the electronic sea and hence may be ferro- or antiferromagnetic, see Ref.[25]). We obtained for all our La-Pr alloys $C$ values in good agreement with those corresponding to their nominal concentration.

[16] See, e.g., P.G. de Gennes, Superconductivity of metals and alloys (Benjamin, N.Y.) 1966, p. 265.

[17] F. Vidal et al., Europhys. Lett. 59, 754 (2002). Let us stress here that as the reduced-temperature range studied in this work is relatively close to $T_{C}(\varepsilon \lesssim 0.1)$ the use of a conventional momentum cutoff [6] would not appreciably modify the theoretical $\Delta M$.

[18] These uncertainties are mainly due to the background extraction. They have been estimated by varying by $10 \%$ the temperature location of the background fitting region.

[19] F. Vidal et al., in Materials science, fundamental properties and future electronic applications of high- $T_{C}$ superconductors, edited by S.L. Drechsler and T.M. Mishonov (Kluwer, Dordrecht) 2001, p. 289; a recently updated version may be found in cond-mat/0510467.

[20] A. Larkin and Yu.N. Ovchinnikov, Sov. Phys. JETP 34, 651 (1972).

[21] Yu.N. Ovchinnikov, S.A. Wolf and V.Z. Kresin, Phys. Rev. B 60, 4329 (1999).

[22] F.S. Bergeret, A.F. Volkov and K.B. Efetov, Europhys Lett. 66, 111 (2004).

[23] F. Soto et al., Phys. Rev. B 70, 060501 (2004).

[24] L.P. Gor'kov and A.I. Rusinov, Sov. Phys. JETP 19, 922 (1964); P. Fulde and R. Ferrel, Phys. Rev. 135, A550 (1964).

[25] M.A. Ruderman and C. Kittel, Phys. Rev. 96, 99 (1954); T. Kasuya, Progr. Teor. Phys. 16, 45 (1956); K. Yosida, Phys. Rev. 106, 893 (1957). 

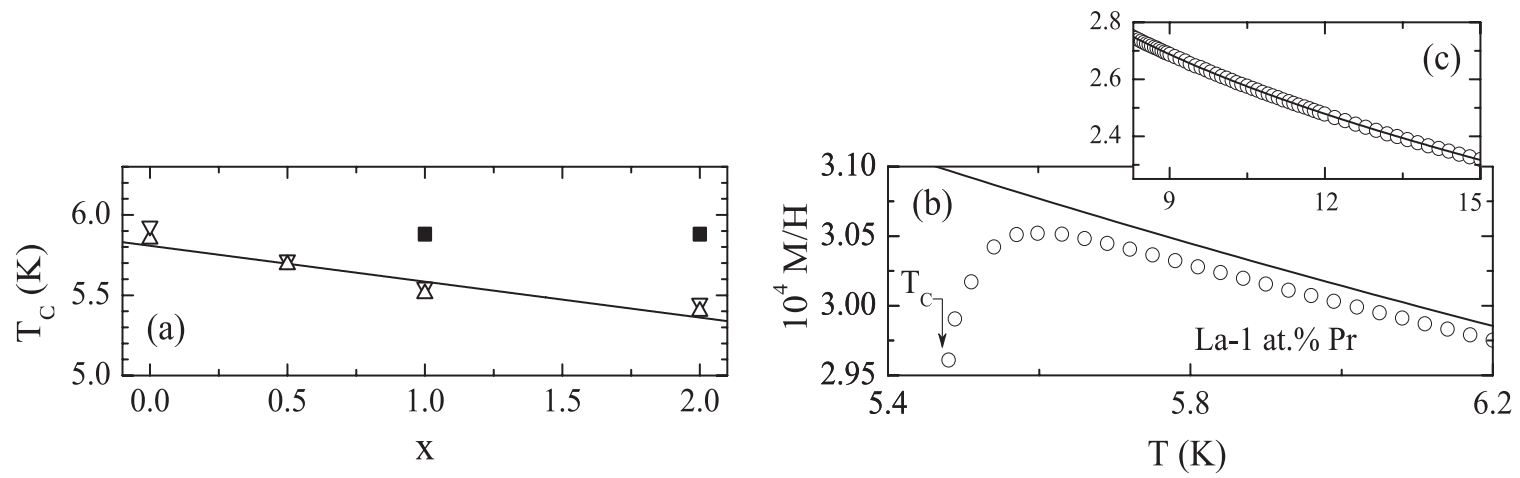

Figure 1: (a) Impurity concentration dependence of the superconducting transition temperature, determined from field-cooled magnetization measurements under $\mu_{0} H=5 \times 10^{-4} \mathrm{~T}$ ( $\triangle$ and $\boldsymbol{\square}$ data) and from the electrical resistivity versus temperature curves $(\nabla$ data). Solid squares correspond to nonmagnetic (Lu) impurities and open symbols to magnetic ( $\mathrm{Pr}$ ) impurities. The solid line is a fit of the Abrikosov and Gor'kov approach. 22 (b) An example, correponding to a La-1 at.\%Pr alloy, of the as-measured magnetization (over $H$ ) versus temperature curves, showing directly the differences between the data and the background $M_{B}(T) / H$ (solid line). These data were obtained under $\mu_{0} H=0.05 \mathrm{~T}$, which is well inside the low-field regime for which $\Delta M$ is linear on the field amplitude. The temperature region shown corresponds well to the reduced temperatures covered in fig. 2(a). (c) Magnetization over $H$ in the background temperature region for the same alloy. 

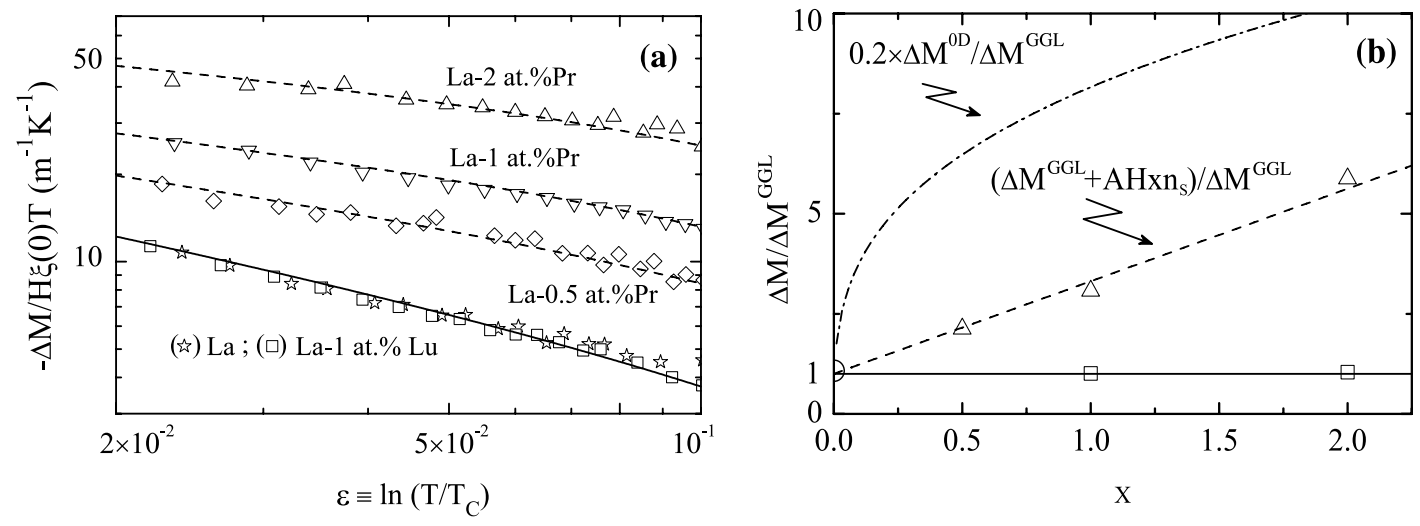

Figure 2: (a) Fluctuation-induced magnetization (over $H \xi(0) T$ ) versus reduced temperature in all the La-Pr alloys studied in this work and also for pure La and a La1 at. $\% \mathrm{Lu}$ alloy. These data were obtained under low magnetic fields, $\mu_{0} H=0.05 \mathrm{~T}$. The covered $\varepsilon$-range covered corresponds well to the accessible experimental window: closer to $T_{C}$ the data are mainly affected by $T_{C}$-inhomogeneities, whereas above $\varepsilon \simeq 0.1$ they are mainly affected by background uncertainties. Outside this $\varepsilon$-region the experimental uncertainties could become even bigger than $100 \%$. The solid line is the GGL prediction, and the dashed lines the are GGL prediction plus a contribution proportional to the superfluid density and magnetic impurity concentration (see main text for details). (b) Experimental fluctuation-induced magnetization, relative to the GGL predictions [5, 6, 8, as a function of impurity content $x$ in pure-La $(\bigcirc), \operatorname{La}-\operatorname{Pr}(\triangle)$ and La-Lu ( $\square)$, at $\varepsilon=0.06$ and $\mu_{0} H=0.05 \mathrm{~T}$. The superconductors without magnetic impurities follow well the GGL approach (solid line), while in those with magnetic impurities $|\Delta M|$ increases, in essence linearly, with $x$. The dashed and dot-dashed lines are explained in the main text. 


\begin{tabular}{lccccccc}
\hline \hline Sample & $\begin{array}{c}T_{C} \\
(\mathrm{~K})\end{array}$ & $\begin{array}{c}\Delta T_{C} \\
(\mathrm{~K})\end{array}$ & $\begin{array}{c}\mu_{0} H_{c 2}(0) \\
(\mathrm{T})\end{array}$ & $\begin{array}{c}\xi(0) \\
(\AA)\end{array}$ & $\kappa\left(T_{C}\right)$ & $\begin{array}{c}\ell \\
(\AA)\end{array}$ & $\begin{array}{c}d_{i m p} \\
(\AA)\end{array}$ \\
\hline La & 5.85 & 0.16 & 0.8 & 200 & 4.4 & 265 & \\
La-0.5 at.\% Pr & 5.69 & 0.12 & 0.9 & 190 & 5.6 & 150 & 19 \\
La-1 at.\% Pr & 5.51 & 0.25 & 0.9 & 180 & 6.0 & 110 & 15 \\
La-2 at.\% Pr & 5.40 & 0.18 & 1.0 & 180 & 6.4 & 75 & 12 \\
La-2 at.\% Lu & 5.88 & 0.26 & 0.9 & 190 & 4.2 & 210 & 12 \\
\hline \hline
\end{tabular}

Table 1: Summary of basic parameters of the La-Pr superconductors studied in this work, obtained from magnetization and electrical resistivity measurements. [14] For comparison, we included also one of the pure La samples and one of the La-Lu alloys. The average distance between impurities $d_{i m p}$ was estimated from the nominal sample compositions. $\ell$ is the mean free path of the normal electrons extrapolated to $T=0 \mathrm{~K}$, and $\kappa\left(T_{C}\right)$ the Ginzburg-Landau parameter at the critical temperature. The uncertainties in $\xi(0), \kappa\left(T_{C}\right)$ and $\ell$ are of about $20 \%$. 